\title{
Automated Brain Tumor Segmentation Using Hsom
}

\author{
Monica. ${ }^{1}$, Santha.P ${ }^{2}$, Dr.C.N.Marimuthu ${ }^{3}$ \\ ${ }^{1}$ Student of ME, Department of ECE, Nandha Engineering College, India \\ ${ }^{2}$ Assistant Professor, Department of ECE, Nandha Engineering College, India \\ ${ }^{3}$ Dean, Department of ECE, Nandha Engineering College, India
}

\begin{abstract}
In this project a forceful segmentation tool for the detection of brain tumour is used to assist clinician and researchers in radio surgery applications. A clustering based approach using hierarchal selforganizing map algorithm is proposed for MR image segmentation. Hierarchal self-organizing map (HSOM) is a dynamically growing neural network model evolves into a hierarchical structure according to the requirements of the input data during an unsupervised training process. This algorithm proved to be exceptionally successful for data visualization applications. HSOM is the extension of the conventional self organizing map which is used to classify the image row by row. This project describe segmentation method consists of two phases. In the first phase, the MRI brain image is acquired from patient database. In that film artifact and noise are removed. In the second phase (MR) image segmentation is to accurately identify the principal tissue structures in these image volumes. Finally the numbers of affected cells are counted using the row and column wise scanning method.
\end{abstract}

Keywords - HSOM, Image analysis, Magnetic Resonance Imaging (MRI) Segmentation, Tumor detection.

\section{Introduction}

The brain is a soft, delicate, non-replaceable and spongy mass of tissue. It is a stable place for patterns to enter and stabilize among each other. A tumour is a mass of tissue that grows out of control of the normal forces that regulates growth. Brain tumour is a group of abnormal cells that grows inside of the brain or around the brain. Tumours can directly destroy all healthy brain cells. It can also indirectly damage healthy cells by crowding other parts of the brain and causing inflammation, brain swelling and pressure within the skull.

Surgical planning, post-surgical assessment, abnormality detection, and many other medical applications require medical image segmentation. In spite of wide number of automatic and semi-automatic image segmentation techniques, they fail in most cases largely because of unknown and irregular noise, inhomogeneity, poor contrast and weak boundaries which are inherent to medical images. MRI and other medical images contain complicated anatomical structures that require precise and most accurate segmentation for clinical diagnosis.

Brain image segmentation from MRI images is complicated and challenging but its precise and exact segmentation is necessary for tumours detection and their classification, edema, haemorrhage detection and necrotic tissues. For early detection of abnormalities in brain parts, MRI imaging is the most efficient imaging technique. Unlike computerized Tomography (CT), MRI image acquisition parameters can be adjusted for generating high contrast image with different gray level for various cases of neuropathology. Therefore, MRI image segmentation stands in the upcoming research limelight in medical imaging arena.

Image Segmentation is an important and challenging factor in the medical image segmentation. Cellular automata (CA) based seeded tumor segmentation method on contrast enhanced T1 weighted magnetic resonance (MR) images, which standardizes the volume of interest (VOI) and seed selection [1]. HSOM is an artificial neural network model which is very successful for data visualization applications where the mapping is done in two-dimensional representation space [2]. Edema is detected along with tumour. T2 MR Image is only required for the segmentation [3].

Segmentation of 3-D tumour structures from magnetic resonance images (MRI), intensity based fuzzy classification of voxels into tumour and background classes are considered. In case of $\mathrm{N}$-dimensional images object and background are considered. Object and background segments may consist of several isolated parts [4][5].Generation of synthetic multi-modal 3D brain MRI with tumour and edema, along with the ground truth is modelled [6]. Cellular automata techniques is found to be a natural tool for image processing due to their local nature and simple parallel computing [7].

\section{Dicom Image}

The Image Processing Toolbox supports writing files in Digital Imaging and Communications in Medicine (DICOM) format, using the dicomwrite function. This converts the dicom image format into JPEG image format so that pre-processing of the image becomes easier. 


\section{Pre-Processing}

The MR image consists of film artifact and labels on the MRI such as patient name, age and marks. Film artifacts are removed using pre-processing. Removal of the artifacts will start from the first row and the first column, the intensity value, greater than that of the threshold value are removed from MRI. The artifact film will have high intensity. These high intensity values of film artifact are removed from MRI brain image. During removal of film artifacts, the images will have salt and pepper noise. Histogram equalization is a spatial domain image enhancement technique that modifies the distribution of the pixels to become more evenly distributed over the available pixel range [8]. In histogram processing, a histogram displays the distribution of the pixel intensity value, mimicking in the probability density function (PDF) for a continuous function. An image that has a uniform PDF will have pixel values at all valid intensities. Therefore, it will show a high contrast image.

Histogram equalization creates a uniform PDF or histogram. This can be accomplished by performing a global equalization that considers all the pixels in the entire image or a local equalization that segments the image into regions [9]. The image is then converted into grey scale image, i.e., 2D image. The grey scale image which consists of pixel intensity between $0-255$ where 0 represents black and 255 represents white.

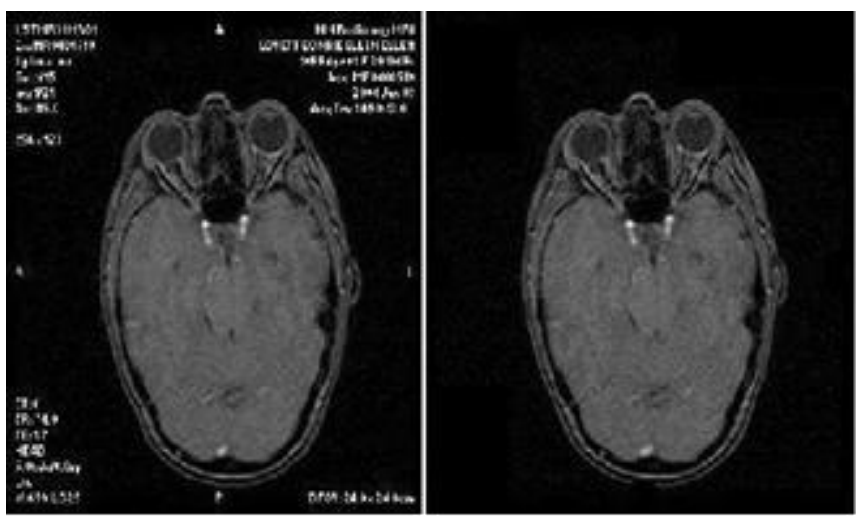

Figure 1 Pre-processing of Input Image

Image enhancement makes the smoothness towards piecewise-homogeneous region and reduces the edgeblurring effects. The information of enhancement using weighted median filter for removing high frequency component.

\section{Hsom Algorithm}

A hierarchical self-organizing map (HSOM) is a type of artificial neural network (ANN). It is an unsupervised learning method to produce a low-dimensional discretized representation of the input space of the training samples, called a map. Hierarchical self-organizing maps are different from other artificial neural networks in the sense that they use a neighbourhood function to preserve the topological properties of the input space. HSOMs operate in two modes: training and mapping. "Training" builds the map using input examples. It is a competitive process, which is also called vector quantization. "Mapping" automatically classifies a new input vector.

The key idea of the hierarchical self-organizing map (HSOM) is to use a hierarchical structure of multiple layers where each layer consists of a number of independent self-organizing maps (SOMs). One SOM is used at the first layer of the hierarchy. This principle is repeated with the third and any further layers of the HSOM.

Segmentation is an important process to extract information from complex medical images. Segmentation has wide application in medical field. Image segmentation techniques can be classified as based on edge detection, region or surface growing, threshold level, classifier such as Hierarchical Self Organizing Map (HSOM), and feature vector clustering or vector quantization. The Trained Vector quantization has proved to be a very effective model for image segmentation process. Vector quantization is a process of portioning $\mathrm{n}$ dimensional vector space into $\mathrm{M}$ regions so as to optimize a criterion function when all the points in each region are approximated by the representation vector Xi associated with that region.

There are two processes involved in the vector quantization: one is the training process which determines the set of codebook vector according to the probability of the input data, the other is the encoding process which assigns input vectors to the code book vectors. Vector quantization process has been implemented in terms of the competitive learning neural network. The importance of SOM for vector quantization is primarily due to the similarity between the competitive learning process employed in the SOM and the vector quantization procedure. The main shortcoming of the SOM is that the number of neural units in the competitive layer needs to be approximately equal to the number of regions desired in the segmented image. 


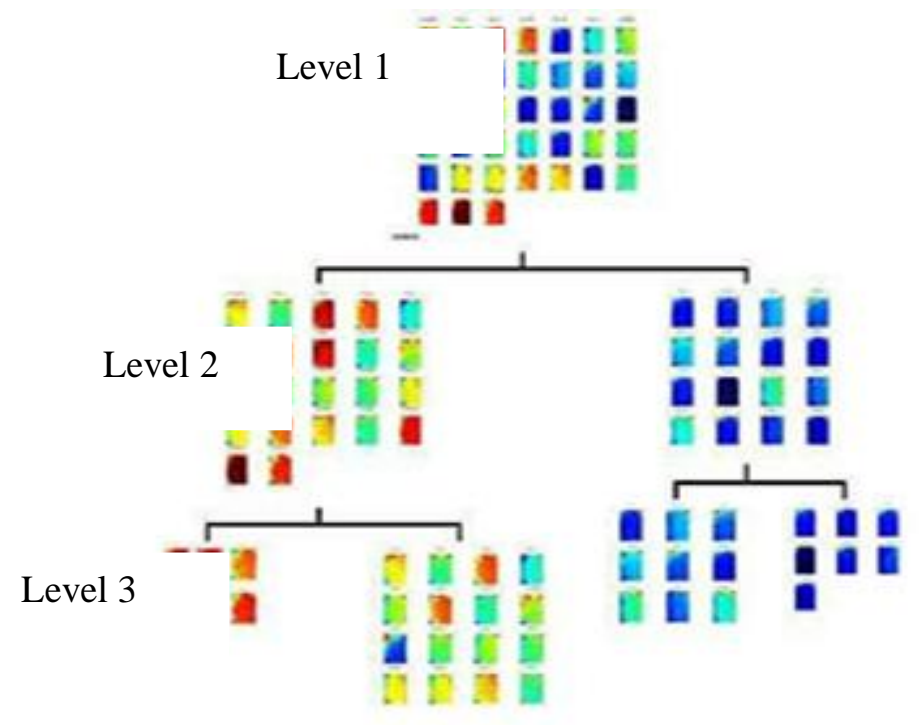

Figure 2 Levels of HSOM

\section{STEPS OF HSOM ALGORITHM}

Step 1: Randomize the map's nodes' weight vectors.

Step 2: Grab an input vector $\mathrm{D}(\mathrm{t})$.

Step 3: Traverse each node in the map

(i) Use the Euclidean distance formula to find the similarity between the input vector and the map's node's weight vector

(ii)Track the node that produces the smallest distance (this node is the best matching unit, BMU)

Step 4: Update the nodes in the neighbourhood of the BMU (including the BMU itself) by pulling them closer to the input vector

$\mathrm{Wv}(\mathrm{s}+1)=\mathrm{Wv}(\mathrm{s})+\Theta(\mathrm{u}, \mathrm{v}, \mathrm{s}) \alpha(\mathrm{s})(\mathrm{D}(\mathrm{t})-\mathrm{Wv}(\mathrm{s}))$

Step 5: Increase $\mathrm{s}$ and repeat from step 2 while $\mathrm{S}<\lambda$

A variant algorithm:

(i)Randomize the map's nodes' weight vectors.

(ii) Traverse each input vector in the input data set.

(a)Traverse each node in the map

(1)Use the Euclidean distance formula to find the similarity between the input vector and the map's node's weight vector

(2)Track the node that produces the smallest distance (this node is the best matching unit, BMU)

(b)Update the nodes in the neighbourhood of the BMU (including the BMU itself) by pulling them closer to the input vector

$\mathrm{Wv}(\mathrm{s}+1)=\mathrm{Wv}(\mathrm{s})+\Theta(\mathrm{u}, \mathrm{v}, \mathrm{s}) \alpha(\mathrm{s})(\mathrm{D}(\mathrm{t})-\mathrm{Wv}(\mathrm{s}))$

(iii) Increase $s$ and repeat from step 2 while $S<\lambda$

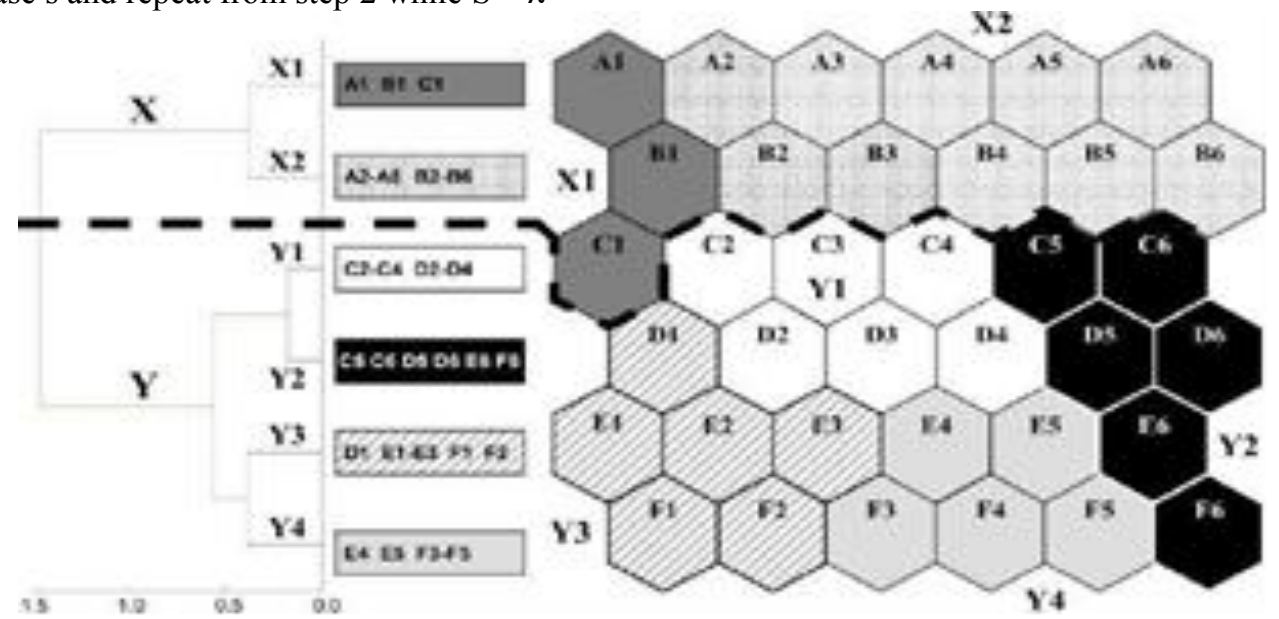

Figure 3 A self-organizing map formed by 36 hexagons 
The 36 hexagons represent neurons as indicated by letter and number. Clusters ( $\mathrm{X}$ and $\mathrm{Y}$ ) and sub clusters (X1, $\mathrm{X} 2$, Y1, Y2, Y3, Y4) of neurons were distinguished on the basis of a hierarchical cluster analysis with the Ward linkage method using Euclidean distance measures. There are two ways to interpret a SOM. Because in the training phase weights of the whole neighbourhood are moved in the same direction, similar items tend to excite adjacent neurons. Therefore, SOM forms a semantic map where similar samples are mapped close together and dissimilar ones apart. This may be visualized by a U-Matrix (Euclidean distance between weight vectors of neighbouring cells) of the SOM. The other way is to think of neuronal weights as pointers to the input space. They form a discrete approximation of the distribution of training samples. More neurons point to regions with high training sample concentration and fewer where the samples are scarce.

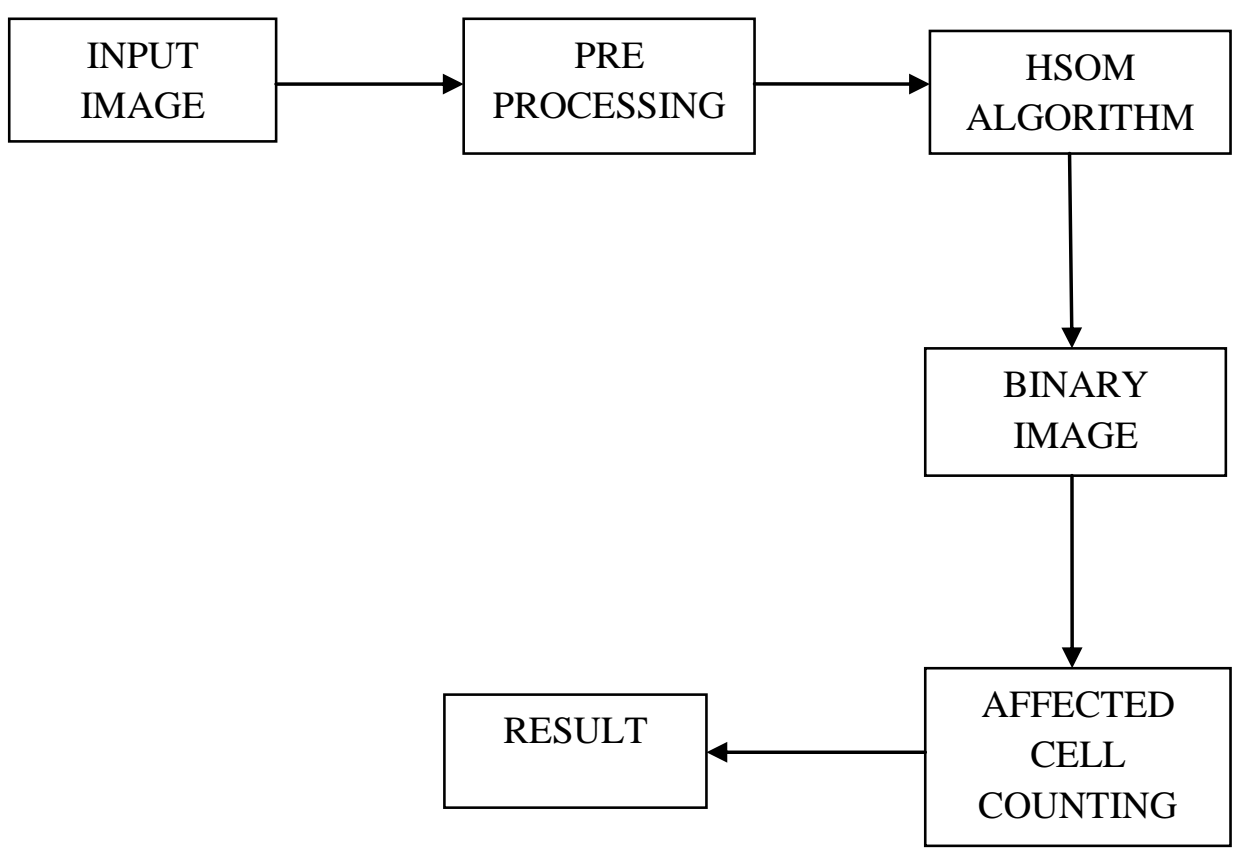

Figure 4 Flow diagram to Detect Tumor

The image is segmented using HSOM algorithm and the conversion of grey scale image into binary image is done. This binary image helps in counting the number of cells affected from the tumour.

V. Results And Analysis

\begin{tabular}{|l|c|c|}
\hline \multicolumn{1}{|c|}{ Input Image } & $\begin{array}{c}\text { No. Of } \\
\text { Affected } \\
\text { Cells }\end{array}$ & $\begin{array}{c}\text { Execution } \\
\text { Time } \\
(\mathrm{sec})\end{array}$ \\
\hline Image 1 & 112 & 1.15441 \\
\hline Image 2 & 117 & 1.31041 \\
\hline Image 3 & 420 & 1.92261 \\
\hline Image 4 & 813 & 2.10601 \\
\hline Image 5 & 1749 & 2.62082 \\
\hline Image 6 & 1808 & 2.73161 \\
\hline Image 7 & 1888 & 2.76981 \\
\hline Image 8 & 3618 & 2.96762 \\
\hline Image 9 & 5162 & 3.71601 \\
\hline
\end{tabular}

Table 1 Simulated Result

Table 1 shows the simulated result of segmentation using HSOM algorithm. The number of cells affected and the time taken to count the cells are also found. The above tabular value is compared with the ground truth values and found to be approximately equal. 


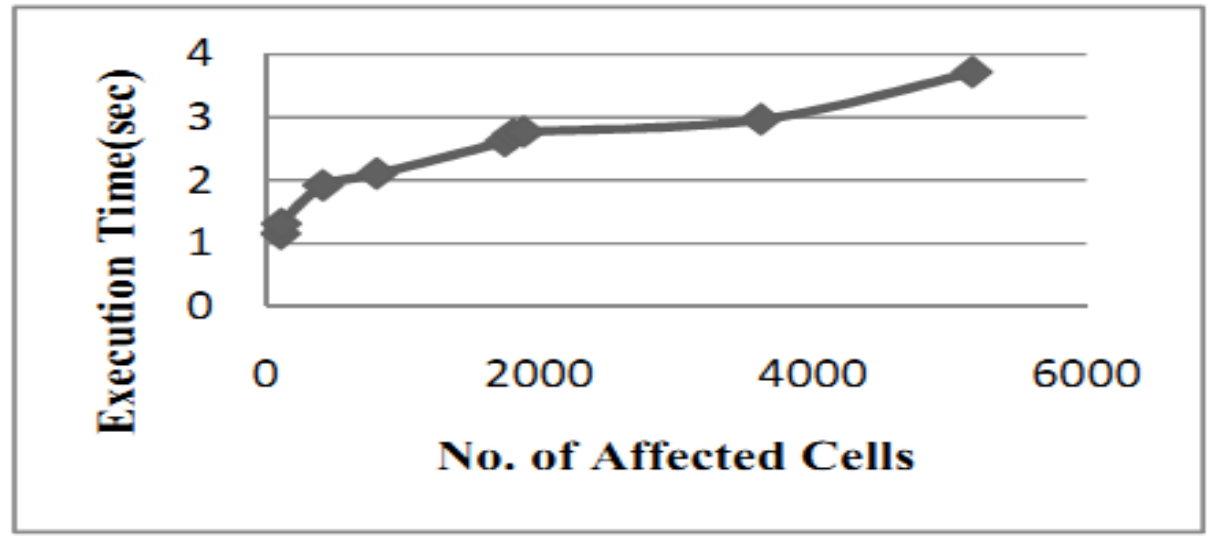

Figure 6 Relations between Number of Affected Cells and Execution Time for Simulated Values SCREEN SHOTS

\section{BRAIN TUMOUR DETECTION}

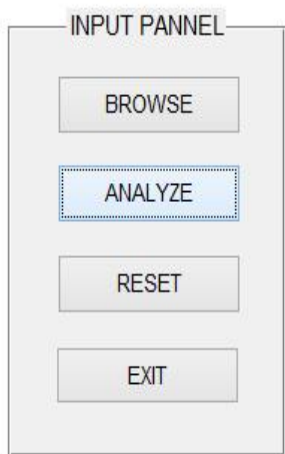

INPUT IMAGE

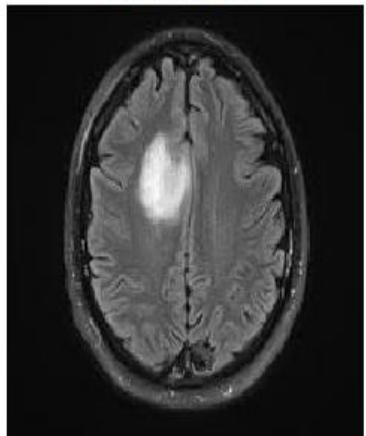

SEGMENTED OUTPUT

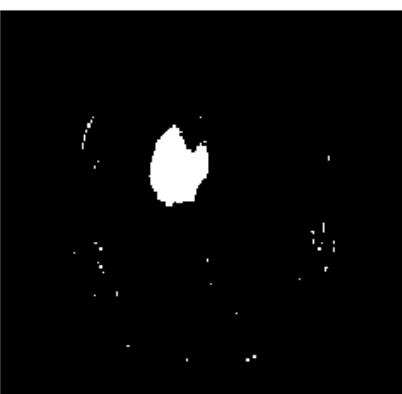

RED CHANNEL

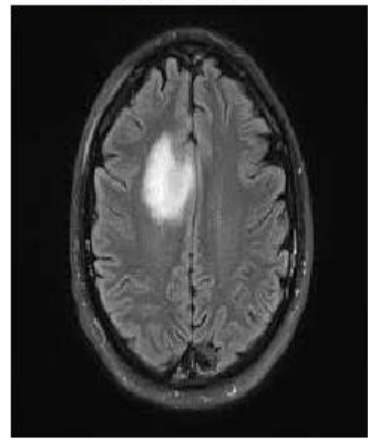

NUMBER OF AFFECTED CELLS

844

SEVERITY ANALYSIS

SEVERE

TIME COMPLEXITY ANALYSIS

SEC

Figure 6 Segmented Output of Image

Figure 6 shows the segmented output of the MR image. The number of cells affected by tumor are 844 and the time taken to analyze is 2.28906 seconds. The segmented tumor is found to be severe.

\section{Conclusion}

The proposed method uses hierarchal self organizing map algorithm using which the red blue and green components are separated. During this separation red component is considered to be best suited for the process of segmentation. With the use of red component segmentation is done and the tumor part is identified. The HSOM algorithm provides the information that how many number of tumor cells are affected. The time taken to detect the tumor cells are also provided using HSOM. The performance of the MR image in terms of execution time and the number of affected cells are detected. 


\section{References}

[1] AndacHamamci, Nadir Kucuk, KutlayKaraman, KayihanEngin, and GozdeUnal,(2012). "Tumor-Cut: Segmentation of Brain Tumors on Contrast Enhanced MR Imagesfor Radiosurgery Applications". IEEE Transactions on Medical Imaging, Vol. 31, No.3.

[2] Fedde van der Lijn, Marleen de Bruijne, Stefan Klein, Tom den Heijer, Yoo Y. Hoogendam, Aad van der Lugt, Monique M. B. Breteler, and Wiro J. Niessen(2012). "Automated Brain Structure Segmentation Based on Atlas Registration and Appearance Models". IEEE Transactions On Medical Imaging, Vol. 31, No. 2

[3] Shanhui Sun, Christian Bauer, and ReinhardBeichel (2012). "Automated 3-D Segmentation of Lungs With Lung Cancer in CT Data Using a Novel Robust Active Shape Model Approach". IEEE Transactions OnMedical Imaging, Vol. 31, No. 2.

[4] Nazem-ZadehM.R, Davoodi-Bojd.E., and Soltanian-ZadehH.,(2011). "Atlasbasedfiber bundle segmentation using principal diffusion directions and spherical harmonic coefficients," NeuroImage, vol. 54, pp. S146-S164.

[5] Gooya, G. Biros, and DavatzikosC., (2011). "Deformable registration of glioma images using em algorithm and diffusion reaction modeling," IEEETrans. Med. Imag., vol. 30, no. 2, pp. 375-390.

[6] Menze,Leemput.K.VLashkari.D,Weber.M.A,. Ayache.N, andGolland.P, (2010)“A generative model for brain tumor segmentation in multimodal images," Med. Image Comput. Comput. Assist. Intervent., vol. 13, pp. 151-159.

[7] Hamamci, Unal.G,.Kucuk.N, and Engin.K., (2010) "Cellular automata segmentation of brain tumors on post contrast MR images," in MICCAI. New York: Springer, pp. 137-146.

[8] Kauffmann and Pich.N., (2010) "Seeded ND medical image segmentation by cellular automaton on GPU," Int. J. Comput. Assist. Radiol. Surg., vol. 5, pp. 251-262.

[9] Bai.X, and Sapiro.G,(2009)., "Geodesic matting: A framework for fast interactive image and video segmentation and matting," Int. J. Comput. Vis., vol. 82, pp. 113-132.

[10] Couprie.C, Grady.L, Najman.L, and Talbot.H. (2009), "Power watersheds:A new image segmentation framework extending graph cuts, randomwalker and optimal spanning forest," in ICCV , pp. 731-738.

[11] Criminisi, Sharp.T, and Blake.A. (2008), “GeoS: Geodesic image segmentation,”inComput. Vis. ECCV, vol. 5302, pp. 99-112

[12] Szeliski.R.,Zabih.R,Scharstein.D, Veksler.O,Kolmogorov.V,Agarwala.A, Tappen.M, and Rother.C, (2008) "A comparative study of energy minimization methods for Markov random fields with smoothness-based priors," IEEE Trans. Pattern Anal. Mach. Intell., vol. 30, pp. 1068-1080.

[13] Sinop and Grady.L. (2007), "A seeded image segmentation framework unifying graph cuts and random walker which yields a new algorithm," in ICCV, pp. 1-8.

[14] Angelini.E.D, Clatz.O, Mandonnet.E, Konukoglu.E, Capelle.L, and Duffau.H(2007), “Glioma dynamics and computational models: A review of segmentation, registration, and in silico growth algorithms and their clinical applications," Curr. Med. Imag. Rev., vol. 3, no. 4, pp.262-276.

[15] Alvino.C, Unal.G, Slabaugh.G, Peny.B, and Fang.T, (2007). "Efficient segmentation based on eikonal and diffusion equations," Int. J. Comput.Math., vol. 84, pp. 1309-1324.

[16] Archip.N, Jolesz.F, and Warfield.S, (2007) "A validation framework for brain tumor segmentation," Acad. Radiol., vol. 14, no. 10 , pp. $1242-1251$. 